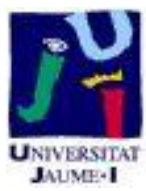

Título artículo / Títol article: The role of forgone opportunities in decision making under risk

Autores / Autors

Barreda Tarrazona, Iván José ; Jaramillo Gutiérrez, Ainhoa ; Navarro Martínez, Daniel ; Sabater Grande, Gerardo

Revista:

Journal of Risk and Uncertainty October 2014, Volume 49, Issue 2

Versión / Versió:

Preprint de l'autor

Cita bibliográfica / Cita

BARREDA-TARRAZONA, Ivan, et al. The role of bibliogràfica (ISO 690): forgone opportunities in decision making under risk. Journal of Risk and Uncertainty, 2014, 49.2: 167-188.

url Repositori UJI:

http://hdl.handle.net/10234/127655 


\section{THE ROLE OF FORGONE OPPORTUNITIES IN DECISION MAKING UNDER RISK}

\section{IVÁN BARREDA-TARRAZONA}

Department of Economics and LEE, University Jaume I, Av. de Vicent Sos Baynat s/n, 12071 Castellón de la Plana, Spain.

\section{AINHOA JARAMILLO-GUTIÉRREZ}

Department of Applied Economics, University of Valencia, Av. Los naranjos, s/n, 46022 Valencia, Spain.

\section{DANIEL NAVARRO-MARTÍNEZ}

Departments of Economics and Psychology, University of Warwick, Coventry CV4 7AL, United Kingdom.

Corresponding author. Tel.: +44 (0)24 765 28388; fax.: +44 (0)24 765 24225. E-mail address: d.navarro-martinez@warwick.ac.uk.

\section{GERARDO SABATER-GRANDE}

Department of Economics and LEE, University Jaume I, Av. de Vicent Sos Baynat s/n, 12071 Castellón de la Plana, Spain.

\section{Acknowledgements}

The authors would like to acknowledge financial support by the Spanish Ministry of Science and Innovation (ECO2008-04636/ECON, and José Castillejo Grants JC2009-16 and JC200700175), the Spanish Ministry of Education (SEJ2007-67204), the Regional Government of Valencia (GV/2007/097 and GVPRE/2008/322), Bancaixa (P11A2006-13, P1 1A2007-06 and Mobility Grants), and the University Jaume I (PREDOC/2005/49). 


\title{
THE ROLE OF FORGONE OPPORTUNITIES IN DECISION MAKING UNDER RISK
}

\begin{abstract}
We present an economic experiment designed to study the role of forgone opportunities in decision making under risk. By facing individuals with a dynamic environment in which their decisions, together with chance, determine their future options, we show that previously faced opportunities influence subsequence choices in predictable ways. Having faced worse options in the past increases significantly individuals' risk aversion and having faced better options reduces it. These patterns are fundamentally at odds with most existing decision theories, including Expected Utility Theory and standard versions of Prospect Theory, the dominant models in economics. Two alternative theoretical accounts are discussed.
\end{abstract}

It seems apparent on an intuitive level that the opportunities we forgo affect our evaluation of the options currently available to us. A good example of this is that individuals constantly evaluate their outcomes relative to "what might have been". Some prominent cases of this kind of behaviour can be found in the psychological literature on counterfactual thinking. For instance, Medvec et al. (1995) showed that silver medallists are generally less happy with their results than bronze medallists. Silver medallists seem to feel closer to the opportunity of getting gold and compare their outcome with it. On the other hand, bronze medallists feel closer to the option of finishing without a medal and compare their achievement with it. These counterfactuals do not affect at all the actual outcomes of the athletes, but they determine how they are evaluated.

Along the same lines, Kahneman and Tversky (1982) faced people with the hypothetical situations of two travellers that missed their flights due to a traffic jam. One of them missed the flight by 30 minutes and the other only by 5 minutes, because his flight had been delayed for 25 minutes. The authors show that people evaluate significantly more negatively the second situation than the first one. Although the actual outcome to be evaluated is the same in both situations (the travellers missed their flights), the opportunity of having arrived on time seems closer in the second case and, consequently, it leads to a stronger positive counterfactual that affects negatively the evaluation of the actual outcome. Other interesting illustrations of the effects of counterfactual thinking can be found, for example, in Johnson 
(1986), Kahneman and Miller (1986), Medvec and Savitsky (1997), Roese (1999) or Epstude and Roese (2008) (see Roese and Olson, 1995, for a review).

Despite these ideas, virtually all existing decision theories assume that the evaluation of the options available to the decision maker is completely independent of previously faced opportunities (unless those opportunities actually affect the decision maker's asset position). This is certainly an assumption of Expected Utility Theory, EUT, (von Neumann and Morgenstern, 1947) and of standard versions of Prospect Theory, PT, (Kahneman and Tversky, 1979; Tversky and Kahneman, 1992), the dominant decision models in economics. Other prominent theories that operate under this assumption include, for example, RankDependent Utility Theory (Quiggin, 1982), Regret Theory (Bell, 1982; Loomes and Sugden, 1982), the Transfer of Attention Exchange (TAX) model (see Birnbaum, 2008), Decision Field Theory (Busemeyer and Townsend, 1993) or static choice heuristics (see, for example, Brandsttater et al., 2006).

In this paper, we present an economic experiment designed to study the role of forgone opportunities in decision making under risk. We face participants with a computerized dynamic decision environment in which their current choices between risky monetary alternatives, together with chance, determine their future options. Thereby, we show that previously faced opportunities affect subsequent economic decisions in predictable ways. Namely, having faced worse options in the past significantly increases individuals' degree of risk aversion and having faced better options reduces it. These findings have wide-ranging implications for economic theory and important potential applications.

We propose two alternative theoretical approaches to accommodate the role of previously faced opportunities in decision making. The first one involves adopting a reference-dependent approach in which the reference point is determined by expectations influenced by previously faced options, along the lines of the models recently proposed by Koszegi and Rabin (2006, 2007, 2009). This approach also links our findings with a recent paper by Post et al. (2008) in which a data set from the "Deal or no Deal" TV game show is used to demonstrate that decision making in the game seems to be path-dependent (that is, affected by previous choice situations). The authors propose a reference-dependent model with a slow-adjusting reference point to account for their findings. As will be explained in Section 1, our experimental design shares some important similarities with the Deal or no Deal game environment.

The second theoretical account we propose can be parsimoniously derived from Stewart et al.'s (2006) Decision by Sampling (DbS) model. DbS is a sequential-sampling decision 
theory, according to which the value assigned to choice options depends on the relative rank that their attributes occupy within relevant comparison samples taken from the current choice environment and from memory. In such a framework, previously faced options systematically affect subsequent decisions and our findings can be straightforwardly explained.

The rest of the paper is organized as follows. In Section 1, our experimental design is explained; in Section 2, the results are presented; in Section 3, our proposed theoretical accounts are discussed; Section 4 concludes.

\section{Experimental Design}

The main goal of our experimental design is to construct a controlled economic decision environment that is both dynamic and risky, in which the choices that individuals make, together with chance, determine the options available to them in the future. That environment will then be used to produce transparent tests of the influence of previously faced opportunities on economic decision making.

With that goal in mind, we created an individual computerized game, which revolves around two different types of assets; one type pays $€ 100$ and the other $€ 0 .{ }^{1}$ Five assets of each type are involved in the game (10 overall). The different assets are represented by transparent numbered boxes displayed on the screen in two separate columns, labelled as good boxes, containing $€ 100$, and bad boxes, containing $€ 0$ (see screenshot from the game in Figure 1). The game consists of a series of rounds, in which subjects have to choose between a sure amount of money offered to them and progressing to the next round. Each time the subject chooses to progress to the next round, one of the remaining assets is automatically lost (that is, removed from the game by the computer) and the next offer is made. If participants reject all the sure amounts of money offered to them throughout the game, they end up getting the final remaining asset as a payoff. Thus, the game consists of a maximum of nine decisions between a sure sum of money and progressing to the next scenario. At the end of the game, subjects were actually paid their corresponding prizes.

\section{[INSERT FIGURE 1 HERE]}

\footnotetext{
${ }^{1}$ The experiment was programmed using Z-Tree, originally developed by Urs Fischbacher (2007) at the University of Zürich.
} 
The sure amounts of money offered to the participants follow always a fixed rule, which is the $80 \%$ of the average value (AV) of the remaining assets. This rule, together with the rest of the game, was thoroughly explained at the beginning of the experimental sessions, and the $\mathrm{AV}$ and the $80 \%$ of the AV of the outstanding boxes were always clearly displayed on the screen. ${ }^{2}$ Moreover, the percentages of remaining good and bad boxes were at all times shown on the screen too. After each decision, participants received additional information on the amounts rejected and the boxes lost thus far.

In essence, we have a dynamic decision environment in which subjects face opportunities, in the form of sums of money, and those opportunities change as a function of subjects' choices and chance.

Finally, to attain a clear picture of the role of forgone opportunities in decision making, we need to generate transparent comparison points in which participants reach exactly the same decisions but having faced different opportunities. To achieve that, we predesigned 8 different elimination sequences of good and bad boxes and assigned subjects randomly to them. Each elimination pattern of good and bad boxes was presented under different boxnumber successions to different subjects. All the sequences were designed to extend over the maximum of nine possible decisions, reaching a standardized final round in which participants faced the exact same choice between a sure amount of $€ 40$ and a 50/50 change of getting either $€ 100$ or $€ 0$. The box removed in that last round, and consequently the payoff received by the participants who did not accept any of the offers, was randomly selected by the computer. In this respect, we simply told subjects that the boxes to be eliminated were randomly determined.

As shown in Table 1, in sequences 1 and 2 (S1 and S2), the elimination of good and bad boxes is alternated, starting with a good box in S1 and with a bad box in S2. In S3 and S4 two eliminations of the same kind are alternated with two of the other, starting with good boxes in S3 and with bad boxes in S4. S5 and S6 consist of three eliminations of the same type followed by three of the other, plus two alternated boxes at the end. S5 begins with good ones and S6 with bad ones. In S7 and S8, four eliminations of the same kind are followed by 4 of the other, starting with good boxes in S7 and with bad ones in S8.

Thus, in all the odd-numbered sequences, subjects always face offers lower or equal to the initial and final $€ 40$, which reach lower levels as the number of the sequence increases. In

\footnotetext{
${ }^{2}$ Written instructions are available from the authors upon request.
} 
the even-numbered sequences, participants always face offers higher or equal to $€ 40$, which get to higher levels in higher-numbered sequences.

\section{[INSERT TABLE 1 HERE]}

The structure of the sequences provides several absolutely transparent comparison points, in which subjects face exactly the same choices but having faced different opportunities. It is especially interesting in this respect that all the subjects who reject the previous offers and get to the final round reach the same decision, but following markedly different paths depending on the sequence.

A total of 265 university students participated in the experiment (slightly over 30 per sequence). It was organized in 8 identical sessions that lasted about one hour each.

It is interesting to note that our decision environment shares some important similarities with the "Deal or No Deal" TV game show. We construct, however, a fully controlled experimental environment that allows for transparent tests of the influence of previously faced opportunities on decision making. Thus, our choice environment is defined by some key features not present in the Deal or no Deal game. For instance, transparent and controlled comparison points to study the role of forgone alternatives, a predefined dynamic environment with a fixed rule to generate choice options, or individual and private decisions.

\section{Results}

To begin with, Table 2 shows a comprehensive descriptive summary of the results obtained in the experiment. The leftmost column contains the number of subjects facing each particular sequence. The rightmost column displays the number of participants remaining in the final round, that is, the number of individuals that rejected all the previous offers and got to the final decision between a sure amount of $€ 40$ and a 50/50 chance of $€ 100$ or $€ 0$. Finally, the third row for each sequence shows the percentage of remaining subjects accepting the sure offer in each specific round of the game. These data completely characterize the behaviour displayed by participants in the game. In other words, the original data set can be completely reconstructed from Table 2 .

[INSERT TABLE 2 HERE] 
As the table shows, between $11 \%$ and $23 \%$ of the subjects, depending on the sequence, accepted the initial offer of $€ 40$ and stopped playing in the first round; between $23 \%$ and $48 \%$ of the participants got to the final round; and between $30 \%$ and $75 \%$ of the subjects that faced the final round accepted the last offer. If we assume for instance, just to illustrate, EUT and the widely-used utility function $u(x)=(x)^{1-r} /(1-r)$, with constant relative risk aversion, the coefficient of relative risk aversion that makes subjects indifferent in the final round between the sure offer of $€ 40$ and the $50 / 50$ chance of $€ 0$ or $€ 100$ is $r=0.24$. This means that, under those assumptions, between $30 \%$ and $75 \%$ of the subjects, with an average of $46.91 \%$, show a coefficient of relative risk aversion greater than 0.24 in the last round. These figures are broadly consistent with previous elicitations of risk attitudes found in the literature under the indicated utility function (see, for example, Holt and Laury, 2002).

Let us now focus on the main issue of how the rates of acceptance are affected by the opportunities faced by subjects during the game, making use of the transparent comparison points generated by our design. The small number of participants accepting the sure offer in each particular round makes it difficult to get statistically significant results by comparing only single sequences to each other. It is straightforward, however, to group sequences together in a meaningful way to get stronger and clearer results. In what follows, we will focus mainly on the final round, where all the sequences get to the exact same choice but having followed different paths.

One clear pattern emerges out of that final stage. Namely, all the sequences in which subjects have only faced offers lower or equal to the initial and final $€ 40$ (the odd-numbered sequences) show higher percentages of acceptance, or in other words higher degrees of risk aversion, than the sequences in which participants have always faced offers higher or equal to $€ 40$ (the even-numbered sequences). In the odd-numbered sequences, the percentages of acceptance range from $47 \%$ to $75 \%$, whereas in the even-numbered sequences they range from $30 \%$ to $43 \%$. As Figure 2 depicts, the difference between the means of the two groups of sequences is $23 \%$.

Note also that this pattern repeats itself in all the transparent comparison points of the game, which include as well round 3 for sequences 1 and 2, round 5 for sequences 1 to 4 and round 7 for sequences 1, 2, 5 and 6. In all those points, the odd-numbered sequences display higher rates of acceptance than the even-numbered ones, with the only exception of sequence 1 in round 5, which shows a percentage slightly below the highest even-numbered one. 


\section{[INSERT FIGURE 2 HERE]}

The statistical significance of these results is verified by a Fisher's exact test comparing odd- and even-numbered sequences in the final round (p-value: 0.02). In other words, the sequences in which subjects have faced offers below or equal to $€ 40$ show significantly higher acceptance rates (or degrees of risk aversion) in the final round that the ones in which participants have faced offers above or equal to €40. A Fisher's exact test comparing the proportions showed by odd- and even-numbered sequences in the first round (p-value: 0.41) confirms that the observed differences do not come from differences in the samples, but are a consequence of the path followed by subjects in the game. The opportunities previously faced are the only difference between the two groups of sequences at the transparent comparison point provided by the final round.

Table 3 shows a logistic regression analysis that provides further insight into this pattern of results. Two separate regressions are presented. First, the probability of acceptance in the final round is regressed on the group of sequences (odd versus even), on the average offer received in each particular sequence and on the interaction term between these two variables. Note that the average offer received completely identifies each individual sequence. Within the odd-numbered sequences, offers reach lower levels (and a lower average) as the number of the sequence increases; within the even-numbered sequences, offers reach higher levels (and a higher average) as the number of the sequence increases. To make the regression results meaningful once the interaction term is introduced, the two main variables have been standardized, subtracting the mean and dividing by the standard deviation. To check for consistency, a second regression is presented without the interaction term in it and without standardizing the variables.

The results obtained from the logistic regression analysis are clear-cut and in line with what has been presented so far. Namely, the probability of acceptance in the final round is significantly higher for the subjects that have previously faced offers below or at the initial and final $€ 40$ (in the odd-numbered sequences) than for the ones that have faced offers above or at $€ 40$ (in the even-numbered sequences). The variable representing the specific average size of the offers is not found to be significant, either generally (see main variable) or within any one of the subgroups (see interaction). ${ }^{3}$

\footnotetext{
${ }^{3}$ The interaction between the two main variables was also analyzed following the method suggested by Ai and Norton (2003) and Norton et al. (2004) for nonlinear models, confirming its non-significance.
} 
[INSERT TABLE 3 HERE]

Overall, the results obtained confirm that previously faced options play a significant role in decision making under risk. In the next section, two alternative theoretical accounts for our findings are suggested and discussed.

\section{Theoretical Accounts}

Two alternative theoretical approaches to account for the role of forgone opportunities in decision making avail themselves. One of them comes from the reference-dependent approach to individual preferences, well-known in economics; the other one comes from the more psychological sampling approach to decision making. Let us discuss them briefly one by one.

The reference-dependent approach.-Reference-dependent approaches to individual preferences have gained widespread success in economics and decision research, especially since the popularization of Prospect Theory (PT). In contrast with more conventional economic approaches, in which the possible outcomes of available choice options are valued in absolute terms (as, for example, in EUT), reference-dependent theories are based on the idea that outcomes are always evaluated relative to some relevant reference point.

Despite their success, a crucial and largely unresolved issue for reference-dependent approaches is how the relevant reference points are determined. In this respect, most researchers have simply made the virtually untested assumption that the reference point is always the current asset position (or status quo) in the relevant domain. It has to be noted however that, although Kahneman and Tversky (1979) suggested current asset position as a relevant aspect of reference points and based most of their analysis on it, such an assumption is not a constituent part of PT. Indeed, the authors explicitly state that "there are situations in which gains and losses are coded relative to an expectation or aspiration level that differs from the status quo.” (p. 286).

Going back to our experiment, it is apparent that standard reference-dependent specifications in which the reference point is just equated with current asset position cannot accommodate the effects of previously faced opportunities found here. Asset position simply 
does not change at all during our game. There are however a small number of recent papers in which reference points are explicitly made dependent on expectations. An important aspect of this kind of approach is that it allows for reference-point effects in contexts in which subjects do not experience actual gains or losses. This provides a well-suited framework to account for the role of forgone opportunities in decision making.

The main models in this area are the ones proposed by Koszegi and Rabin (2006, 2007, 2009). Another interesting example, more similar to the model explained here, can be found in Post et al. (2008). ${ }^{4}$ Below we present a simpler model, based on the idea that reference points depend on expectations determined by previously faced options, to illustrate how this approach can easily account for the type of effects of forgone opportunities found in our experiment.

Let us assume that the reference-dependent value of a specific lottery $L_{j}$, designated as $R D V\left(L_{j}\right)$, is determined by the following expression:

$$
R D V\left(L_{j}\right)=\sum_{i=1}^{n} p_{i} v\left(x_{i}\right)
$$

where $x_{i}$ denotes a specific outcome within the lottery and $p_{i}$ the probability associated to it. $v\left(x_{i}\right)$ is a reference-dependent value function over outcomes, defined by

$$
v\left(x_{i}\right)=\left\{\begin{array}{ll}
-\lambda\left(R P-x_{i}\right)^{\alpha} & \text { if } x_{i}<R P \\
\left(x_{i}-R P\right)^{\alpha} & \text { if } x_{i} \geq R P
\end{array},\right.
$$

where $R P$ is the reference point, $\lambda$ (normally greater than 1 ) is a loss aversion parameter and $\alpha$ is a parameter determining the curvature of the value function. This specification is essentially the one normally used for cumulative PT (Tversky and Kahneman, 1992), but without its characteristic probability weighting, which has been removed here for simplicity. All the explanations given in this section hold under the usual probability weighting functions. This specification could also be viewed as a reference-dependent EUT representation.

\footnotetext{
${ }^{4}$ It should be noted that the "disappointment” theories by Bell (1985) and Loomes and Sugden (1986) were actually the first ones to make reference points explicitly dependent on expectations. In these models, however, expectations do not depend on previously faced situations, but just on the possible outcomes of the alternatives currently available.
} 
Additionally, instead of associating the reference point with the current asset position, let us assume that $R P$ is determined by the following expectation:

$$
R P=O_{n}+\delta\left(\frac{\sum_{k=1}^{n-1} O_{k}}{n}-O_{n}\right)
$$

where $\left\{O_{1}, \ldots, O_{n}\right\}$ represent the offers faced during the game, $O_{n}$ being the current one, and $\delta$ is a (non-negative) parameter determining the weight of previous offers in the present reference point. With $\delta=0, R P$ simply equals $O_{n}$, the current offer, and it is not influenced at all by previously faced options. With $\delta>0, R P$ is an expectation determined jointly by the current offer and the average of the previous offers faced during the game. The higher the average of the offers previously faced, the higher is the expectation that determines the reference point. ${ }^{5}$

It is straightforward that under this specification all the odd-numbered sequences in our experiment, in which subjects face always offers below or equal to the initial and final $€ 40$, result in reference points below the current offer in the final period. On the contrary, all the even-numbered sequences, in which subjects face only offers above or equal to $€ 40$, result in reference points above the current offer in the final period. This establishes a clear difference between these two groups of sequences that leads easily to the main results obtained in the experiment. Namely, that the odd-numbered sequences show significantly higher acceptance rates than the even-numbered ones. Figure 3 illustrates this point.

The three different plots in Figure 3 show how changes in the reference point affect the distance in terms of value (or $R D V$ ) between the two alternatives in the final choice, for three different sets of parameters. The alternative offering a 50/50 chance of $€ 100$ or $€ 0$ is labelled $L_{R}$ (for risky lottery); the sure offer of $€ 40$ is labelled $L_{S}$ (for safe lottery). The vertical axes

\footnotetext{
${ }^{5}$ Other simple variants of this model could include, for example, having all the possible outcomes previously faced (not only the offers) affecting the expectation that determines the reference point; having the median of the amounts previously faced (instead of the average) influencing the reference point; using the average or the median of all the possible outcomes in the current choice (instead of simply the current offer) as the default reference point; or assigning different weights to different periods. Such modifications would allow the model just described to be easily applied to many other decision environments. All the main results discussed here hold for all these variants of the model.
} 
represent $R D V\left(L_{R}\right)-R D V\left(L_{S}\right)$. The first plot depicts the case of risk aversion $(\alpha<1)$ and no loss aversion $(\lambda=1)$. The second plot shows the case of both risk aversion $(\alpha<1)$ and loss aversion $(\lambda>1)$. Finally, the third plot illustrates the case no risk aversion $(\alpha=1)$ and loss aversion $(\lambda>1)$. The specific degrees of risk aversion and loss aversion chosen, $\alpha=0.8$ and $\lambda$ $=2$, are very similar to the ones usually found in implementations of cumulative PT. Any other reasonable parameters result in essentially the same patterns.

\section{[INSERT FIGURE 3 HERE]}

The initial plot demonstrates that, under risk aversion, movements of the reference point downwards from the sure offer of 40 , as the ones found in the odd-numbered sequences, result in a reduced $R D V\left(L_{R}\right)-R D V\left(L_{S}\right)$ and consequently in higher percentages of acceptance of the sure offer $\left(L_{S}\right)$. This is represented by the lower convex segment of the graph. On the contrary, movements of the reference point upwards from 40, as the ones found in the evennumbered sequences, produce a larger $R D V\left(L_{R}\right)-R D V\left(L_{S}\right)$ and therefore lower rates of acceptance. This corresponds to the higher concave segment of the graph. Overall, this is exactly the pattern found in our experiment.

The second plot illustrates that, under both risk aversion and loss aversion, the behaviour of $R D V\left(L_{R}\right)-R D V\left(L_{S}\right)$ for movements of $R P$ upwards from 40 is essentially the same as in the first plot, but the result of movements below 40 tends to reverse. Note however that that reversion results in a much weaker pattern than the one found above 40 . Consequently, again under this specification, which is very much in line with the ones usually employed for cumulative PT, odd-numbered sequences are predicted to show significantly higher percentages of acceptance than even-numbered ones.

Finally, the third plot depicts the case of loss aversion without risk aversion, which is quite uncommon in the literature. Under such a specification, movements of the reference point away from the current offer (40 in the final round), in any direction, result in a larger $R D V\left(L_{R}\right)-R D V\left(L_{S}\right)$ and consequently in lower rates of acceptance. ${ }^{6}$ This pattern together with the one shown in the first plot determines the shape of the plot in the middle.

\footnotetext{
${ }^{6}$ Note however that, even in this case, assuming a lower $\delta$ for movements below the current offer than for movements above it would result in the pattern found in our experiment. Such an assumption seems justified by the idea that people adapt easier to good outcomes than to bad ones.
} 
On the whole, Figure 3 clearly demonstrates that the kind of reference-dependent model proposed, in which the reference point depends on expectations influenced by previously faced options, can effectively account for the type of effects of forgone opportunities identified in the present paper.

Decision by sampling.-Decision by Sampling (DbS, Stewart et al., 2006) is a general theory of decision making based on the idea that individuals make choices by sequentially comparing the attributes of available choice options to samples of similar attributes found in the current choice environment or in memory. Favourable comparisons increase the value assigned to an alternative and the alternative with the highest value is chosen. In such a process, the attributes of a particular choice option contribute to its value according to the relative rank they occupy within the comparison attributes found in the relevant sample. Specifically, a higher relative rank means a higher rate of favourable comparisons and consequently a higher value. This approach provides a remarkably useful framework to account for a wide variety of context effects in decision making (see Stewart, 2009). A closed mathematical formulation of DbS is presented in Stewart and Simpson (2008) and Stewart (2009). The details of it are beyond the scope of the present paper.

DbS provides a parsimonious and effective explanation for our findings. Under the single straightforward assumption that the offers faced in previous rounds remain in subjects' memories during the game, it is apparent that, in the odd-numbered sequences, the relative rank of the final offer of $€ 40$ within the sample of comparison attributes is considerably increased by the offers below $€ 40$ previously faced. On the contrary, in the even-numbered sequences, the relative rank of that offer is notably decreased by the offers above $€ 40$ faced before. Consequently, the final offer will be assigned a significantly higher value in all the odd-numbered sequences than in any of the even-numbered ones. As a result, the percentages of acceptance in the odd-numbered sequences are expected to be significantly higher. This is exactly the pattern identified in our experiment.

\section{Conclusions}

We have shown that previously faced opportunities play a significant role in economic decision making under risk, affecting in predictable ways the evaluation of subsequent choice 
options. Specifically, having faced worse (better) opportunities in the past produces significantly higher (lower) degrees of risk aversion in later decisions. These patterns are fundamentally at odds with most existing decision models (for instance, Expected Utility Theory, standard versions of Prospect Theory, Rank-Dependent Utility Theory, Regret Theory, the TAX model, Decision Field Theory or static choice heuristics) and, consequently, have wide-ranging implications for economic theory. Our results demonstrate that economic decision making should be understood and modelled in a dynamic framework in which the opportunities available to decision makers determine how subsequent choice options are evaluated. These findings also have important potential applications. Generally, they suggest that making certain opportunities available to decision makers will influence the way they evaluate subsequent choice options they encounter in predictable ways. They also provide guidance on how alternatives will be evaluated depending on the opportunities faced before. All these issues certainly merit further investigation in future research.

We have suggested two alternative parsimonious theoretical approaches to the role of previously faced opportunities in decision making. One of them involves constructing reference-dependent models in which reference points are determined by expectations influenced by previously faced options. The other one implies a stronger departure from conventional economic approaches and involves adopting a theoretical framework in which the value assigned to choice options depends on the relative rank of their attributes within relevant comparison samples taken from the current choice environment and from memory.

\section{References}

Ai, C., Norton, E.C. (2003). 'Interaction terms in logit and probit models', Economic Letters, vol. 80, pp. 123-129.

Bell, D.E. (1982). 'Regret in decision making under uncertainty’, Operations Research, vol. 30, pp. 961-981.

Bell, D.E. (1985). 'Disappointment in decision making under uncertainty', Operations Research, vol. 33, pp. 1-27.

Birnbaum, M.H. (2008). 'New paradoxes of risky decision making', Psychological Review, vol. 115, pp. 463-501. 
Brandstatter, E., Gigerenzer, G. and Hertwig, R. (2006). 'The priority heuristic: Making choices without trade-offs', Psychological Review, vol. 113, pp. 409-432.

Busemeyer, J.R. and Townsend, J.T. (1993). 'Decision field theory: A dynamic-cognitive approach to decision making in an uncertain environment', Psychological Review, vol. 100, pp. 432-459.

Epstude, K. and Roese, N.J. (2008). 'The functional theory of counterfactual thinking', Personality and Social Psychology Review, vol. 12, pp. 168-192.

Fischbacher, U. (2007). 'Z-Tree: Zurich Toolbox for Readymade Economic Experiments. Experimenter’s manual', Experimental Economics, vol. 10, pp. 171-178.

Holt, C.A. and Laury, S.K. (2002). 'Risk aversion and incentive effects in lottery choices', American Economic Review, vol. 92, pp. 1644-1655.

Johnson, J.T. (1986). 'The knowledge of what might have been: Affective and attributional consequences of near outcomes', Personality and Social Psychology Bulletin, vol. 12, pp. $51-6$.

Kahneman, D. and Miller, D.T. (1986). 'Norm Theory: Comparing reality to its alternatives', Psychological Review, vol. 93, pp. 136-153.

Kahneman, D. and Tversky, A. (1979). 'Prospect Theory: An analysis of decision under risk', Econometrica, vol. 47, pp. 263-291.

Kahneman, D. and Tversky, A. (1982). 'The simulation heuristic', in (D. Kahneman, P. Slovic and A. Tversky, eds.), Judgment under Uncertainty: Heuristics and Biases, New York: Cambridge University Press.

Koszegi, B. and Rabin, M. (2006). 'A model of reference-dependent preferences', Quarterly Journal of Economics, vol. 121, pp. 1133-1165.

Koszegi, B. and Rabin, M. (2007). 'Reference-dependent risk attitudes', American Economic Review, vol. 97, pp. 1047-1073.

Koszegi, B. and Rabin, M. (2009). 'Reference-dependent consumption plans', American Economic Review, vol. 99, pp. 909-936.

Loomes, G. and Sugden, R. (1982). 'Regret Theory: An alternative theory of rational choice under uncertainty', The Economic Journal, vol. 92, pp. 805-824.

Loomes, G. and Sugden, R. (1986). 'Disappointment and dynamic consistency in choice under uncertainty', Review of Economic Studies, vol. 53, pp. 271-282. 
Medvec, V.H., Madey, S.F. and Gilovich, T. (1995). 'When less is more: Counterfactual thinking and satisfaction among Olympic medallists', Journal of Personality and Social Psychology, vol. 69, pp. 603-610.

Medvec, V. H. and Savitsky, K. (1997). 'When doing better means feeling worse: The effects of categorical cutoff points on counterfactual thinking and satisfaction', Journal of Personality and Social Psychology, vol. 72, pp. 1284-1296.

Norton, E.C., Wang, H. and Ai, C. (2004). 'Computing interaction effects and standard errors in logit and probit models', The Stata Journal, vol. 4, pp. 154-167.

Post, T., van den Assem, M.J., Baltussen, G. and Thaler, R.H. (2008). 'Deal or No Deal? Decision making under risk in a large-payoff game show', American Economic Review, vol. 98, pp. 38-71.

Quiggin, J.C. (1982). 'A theory of anticipated utility', Journal of Economic Behavior and Organization, vol. 3, pp. 323-343.

Roese, N.J. (1999). 'Counterfactual thinking and decision making', Psychonomic Bulletin and Review, vol. 6, pp. 570-578.

Roese, N. J. and Olson, J.M. (1995). What Might Have Been: The Social Psychology of Counterfactual Thinking, Hillsdale, NJ: Erlbaum.

Stewart, N. (2009). 'Decision by sampling: The role of the decision environment in risky choice’, Quarterly Journal of Experimental Psychology, vol. 62, pp. 1041-1062.

Stewart, N., Chater, N. and Brown, G.D.A. (2006). 'Decision by sampling', Cognitive Psychology, vol. 53, pp. 1-26.

Stewart, N. and Simpson, K. (2008). 'A decision-by-sampling account of decision under risk', in (N. Chater and M. Oaksford, eds.), The Probabilistic Mind: Prospects for Bayesian Cognitive Science, Oxford, UK: Oxford University Press.

Tversky, A. and Kahneman, D. (1992). 'Advances in prospect theory: Cumulative representation of uncertainty', Journal of Risk and Uncertainty, vol. 5, pp. 297-323.

von Neumann, J. and Morgenstern, O. (1944). The Theory of Games and Economic Behavior, Princeton: Princeton University Press. 


\section{Tables and figures}

Table 1

Structure of the 8 Sequences* +

\begin{tabular}{llrrrrrrrrr}
\hline & Round & 1 & 2 & 3 & 4 & 5 & 6 & 7 & 8 & 9 \\
\hline \hline S1 & Offer & $\mathbf{4 0}$ & 35.56 & $\mathbf{4 0}$ & 34.29 & $\mathbf{4 0}$ & 32 & $\mathbf{4 0}$ & 26.67 & $\mathbf{4 0}$ \\
& Box removed & G & B & G & B & G & B & G & B & Random \\
\hline S2 & Offer & $\mathbf{4 0}$ & 44.45 & $\mathbf{4 0}$ & 45.72 & $\mathbf{4 0}$ & 48 & $\mathbf{4 0}$ & 53.34 & $\mathbf{4 0}$ \\
& Box removed & B & G & B & G & B & G & B & G & Random \\
\hline S3 & Offer & $\mathbf{4 0}$ & 35.56 & 30 & 34.29 & $\mathbf{4 0}$ & 32 & 20 & 26.67 & $\mathbf{4 0}$ \\
& Box removed & G & G & B & B & G & G & B & B & Random \\
\hline S4 & Offer & $\mathbf{4 0}$ & 44.45 & 50 & 45.72 & $\mathbf{4 0}$ & 48 & 60 & 53.34 & $\mathbf{4 0}$ \\
& Box removed & B & B & G & G & B & B & G & G & Random \\
\hline S5 & Offer & $\mathbf{4 0}$ & 35.56 & 30 & 22.86 & 26.67 & 32 & $\mathbf{4 0}$ & 26.67 & $\mathbf{4 0}$ \\
& Box removed & G & G & G & B & B & B & G & B & Random \\
\hline S6 & Offer & $\mathbf{4 0}$ & 44.45 & 50 & 57.15 & 53.34 & 48 & $\mathbf{4 0}$ & 53.34 & $\mathbf{4 0}$ \\
& Box removed & B & B & B & G & G & G & B & G & Random \\
\hline S7 & Offer & $\mathbf{4 0}$ & 35.56 & 30 & 22.86 & 13.34 & 16 & 20 & 26.67 & $\mathbf{4 0}$ \\
& Box removed & G & G & G & G & B & B & B & B & Random \\
\hline S8 & Offer & $\mathbf{4 0}$ & 44.45 & 50 & 57.15 & 66.67 & 64 & 60 & 53.34 & $\mathbf{4 0}$ \\
& Box removed & B & B & B & B & G & G & G & G & Random \\
\hline$*$ * & Good box (C100); B & B B & box (60) & & & & & &
\end{tabular}

${ }^{*} \mathrm{G}=$ Good box (€100); B = Bad box (€0).

†Red colour designates offers under $€ 40$; blue colour offers above $€ 40$. 
Table 2

Descriptive Summary of Results

\begin{tabular}{|c|c|c|c|c|c|c|c|c|c|c|c|}
\hline & Round & 1 & 2 & 3 & 4 & 5 & 6 & 7 & 8 & 9 & $\begin{array}{l}n \text { last } \\
\text { round }\end{array}$ \\
\hline S1 & Offer & 40 & 35.56 & 40 & 34.29 & 40 & 32 & 40 & 26.67 & 40 & \multirow{3}{*}{16} \\
\hline \multirow[t]{2}{*}{$n=36$} & Box rem. & G & B & G & B & G & B & G & B & Random & \\
\hline & \% accept. & 11.11 & 3.13 & 9.68 & 7.14 & 7.69 & 8.33 & 22.73 & 5.88 & 75 & \\
\hline$S 2$ & Offer & 40 & 44.45 & 40 & 45.72 & 40 & 48 & 40 & 53.34 & 40 & \multirow{3}{*}{13} \\
\hline \multirow[t]{2}{*}{$n=35$} & Box rem. & B & G & B & G & B & G & B & G & Random & \\
\hline & \% accept. & 22.86 & 14.81 & 0 & 8.7 & 0 & 14.29 & 5.56 & 23.53 & 30.77 & \\
\hline S3 & Offer & 40 & 35.56 & 30 & 34.29 & 40 & 32 & 20 & 26.67 & 40 & \multirow{3}{*}{13} \\
\hline \multirow[t]{2}{*}{$n=35$} & Box rem. & G & $\mathrm{G}$ & B & B & G & G & B & B & Random & \\
\hline & \% accept. & 11.43 & 3.23 & 13.33 & 3.85 & 24 & 0 & 21.05 & 13.33 & 61.54 & \\
\hline S4 & Offer & 40 & 44.45 & 50 & 45.72 & 40 & 48 & 60 & 53.34 & 40 & \multirow{3}{*}{13} \\
\hline \multirow[t]{2}{*}{$n=34$} & Box rem. & B & B & G & G & B & B & G & G & Random & \\
\hline & \% accept. & 17.65 & 0 & 17.86 & 0 & 8.7 & 19.05 & 23.53 & 0 & 38.46 & \\
\hline S5 & Offer & 40 & 35.56 & 30 & 22.86 & 26.67 & 32 & 40 & 26.67 & 40 & \multirow{3}{*}{8} \\
\hline \multirow[t]{2}{*}{$n=32$} & Box rem. & G & G & G & B & B & B & G & B & Random & \\
\hline & \% accept. & 15.63 & 3.7 & 3.85 & 16 & 4.76 & 20 & 25 & 33.33 & 50 & \\
\hline S6 & Offer & 40 & 44.45 & 50 & 57.15 & 53.34 & 48 & 40 & 53.34 & 40 & \multirow{3}{*}{10} \\
\hline \multirow[t]{2}{*}{$n=32$} & Box rem. & B & B & B & G & G & G & B & G & Random & \\
\hline & \% accept. & 15.63 & 3.7 & 11.54 & 13.04 & 10 & 0 & 16.67 & 33.33 & 30 & \\
\hline S7 & Offer & 40 & 35.56 & 30 & 22.86 & 13.34 & 16 & 20 & 26.67 & 40 & \multirow{3}{*}{5} \\
\hline \multirow[t]{2}{*}{$n=31$} & Box rem. & G & G & G & $\mathrm{G}$ & B & B & B & B & Random & \\
\hline & \% accept. & 22.58 & 0 & 4.17 & 0 & 4.35 & 0 & 13.64 & 21.05 & 46.67 & \\
\hline S8 & Offer & 40 & 44.45 & 50 & 57.15 & 66.67 & 64 & 60 & 53.34 & 40 & \multirow{3}{*}{7} \\
\hline \multirow[t]{2}{*}{$n=30$} & Box rem. & B & B & B & B & G & G & G & G & Random & \\
\hline & \% accept. & 20 & 4.17 & 30.43 & 12.5 & 14.29 & 33.33 & 0 & 12.5 & 42.86 & \\
\hline
\end{tabular}

Table 3

Logistic Regression Results for Probability of Acceptance, Final Round

\begin{tabular}{r|rrrr}
\hline Specification & Estimate & Std. error & z value & p value \\
\hline \hline odd-even (oe) & -2.271 & 1.092 & -2.080 & $\mathbf{0 . 0 3 8}$ \\
average offer (ao)* & 0.994 & 0.640 & 1.552 & 0.121 \\
oe*ao & -0.568 & 1.052 & -0.539 & 0.590 \\
intercept & 1.220 & 0.617 & 1.980 & $\mathbf{0 . 0 4 8}$ \\
\hline
\end{tabular}

*The variable "ao" has been standardized in this specification, subtracting the mean and dividing by the standard deviation.

\begin{tabular}{r|rrrr}
\hline Specification & Estimate & Std. error & z value & p value \\
\hline \hline odd-even (oe) & -2.459 & 1.036 & -2.373 & $\mathbf{0 . 0 1 7 7}$ \\
average offer (ao) & 0.098 & 0.063 & 1.556 & 0.1198 \\
intercept & -2.766 & 2.043 & -1.354 & 0.1757 \\
\hline
\end{tabular}




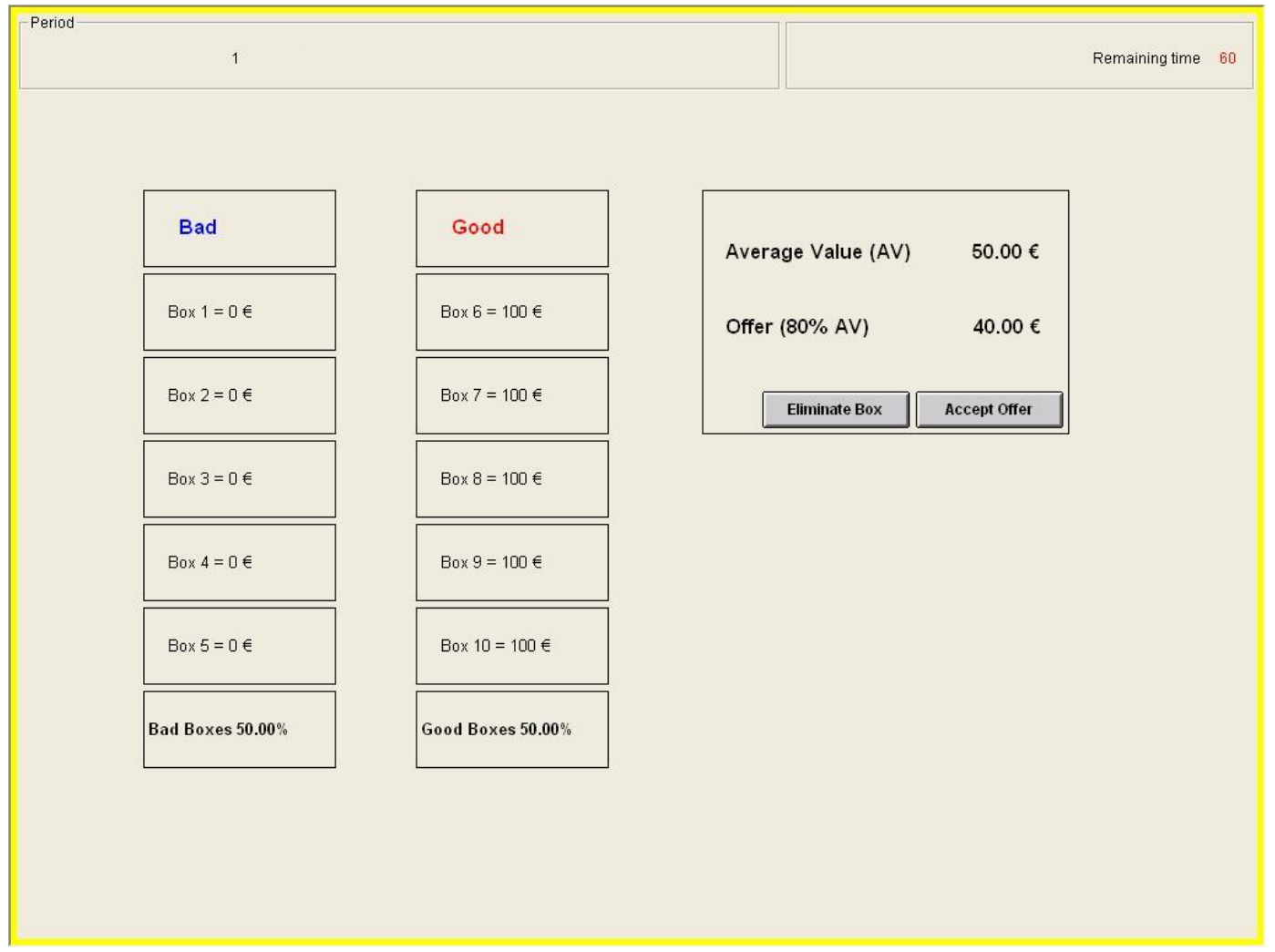

Fig. 1. Screenshot from the Game

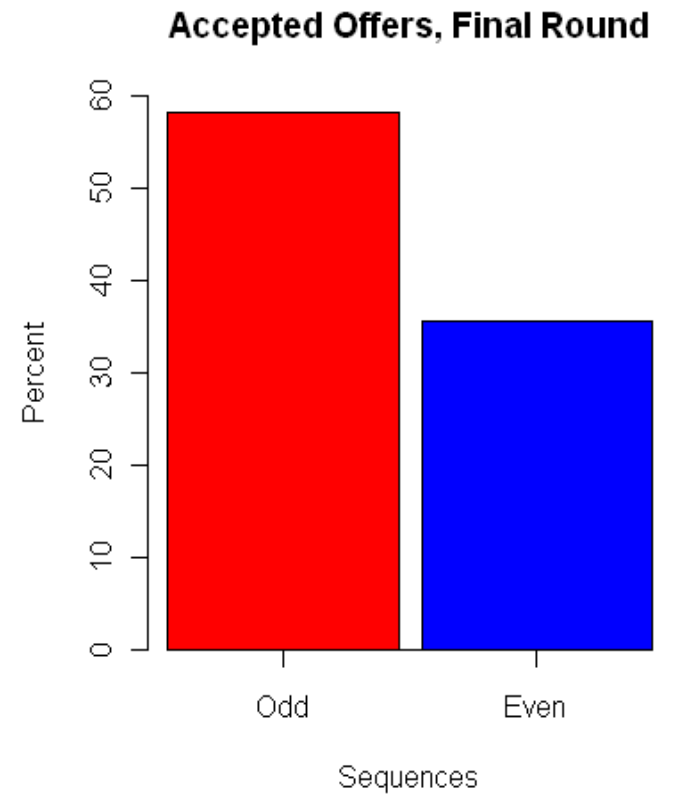

Fig. 2. Average Percentages of Acceptance in the Final Round in Odd-and Even-Numbered Sequences 

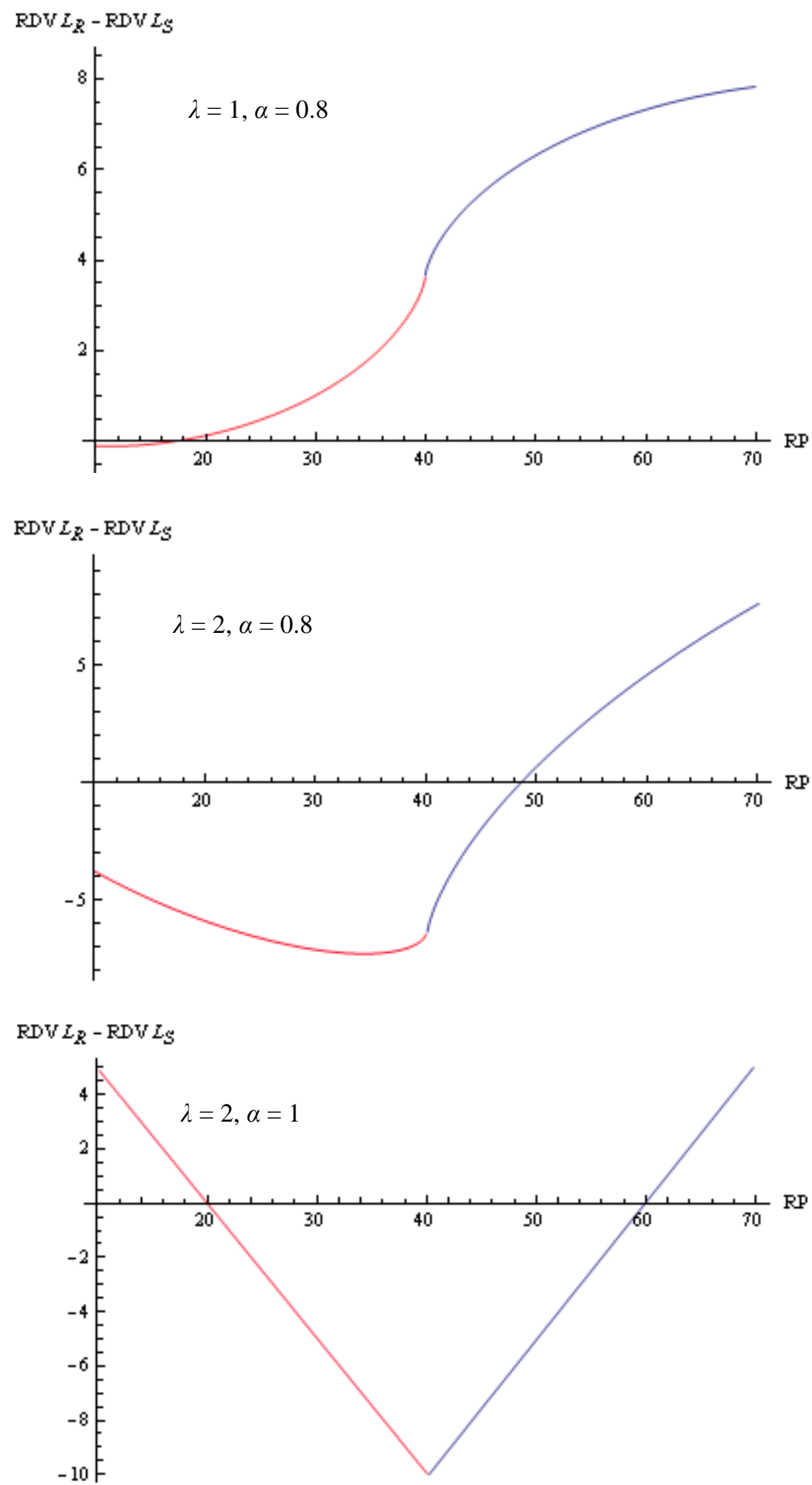

Fig. 3. Effects of Changes in the Reference Point (RP) on $R D V\left(L_{R}\right)-R D V\left(L_{S}\right)$ 